\title{
Fracture Mechanisms of a Thin Elastic Plastic Laminate
}

\author{
C. Bjerkén, S. Kao-Walter and P. Ståhle \\ Division of Materials Mechanics \\ Malmö University \\ SE205 06 Malmö \\ Sweden \\ Phone: +46(0)40-6657688 FAX: +46(0)40-6657135 \\ E-mail: pers@ts.mah.se
}

\begin{abstract}
The fracture toughness of a polymer-metal laminate composite is obtained by mechanical testing of a specimen containing a pre-crack. The laminate is a material used for packaging. It consists of a thin aluminium foil and a polymer coating. A centre cracked panel test geometry is used. Each of the layers forming the laminate is also tested separately. The result is compared with the measured fracture strength of the individual layers. It is observed that the load carrying capacity increases dramatically for the laminate. At the strain when peak load is reached for the laminate only aluminium is expected to carry any substantial load because of the low stiffness of the LDPE. However, the strength of the laminate is almost twice the strength of the aluminium foil. The reason seems to be that the aluminium forces the polymer to absorb large quantities of energy at small nominal strain. The toughness compares well with the accumulated toughness of all involved layers. Possible fracture of the interface between the layers is discussed.
\end{abstract}

\section{Introduction}

Liquid food packages are often made of packaging materials consisting of different material layers to fulfil several requirements of the package. It is very important to ensure that every layer maintains its function during the forming, filling and transportation processes. As an example here a liquid food packaging material is considered. This is a laminate consisting of LDPE (Low Density Polyethylene) and an aluminium foil. Several studies of different mechanical properties of these materials have been performed [1-5]. It was found in [2] that aluminium foil and LDPE laminated together provide significantly higher stress and strain at fracture as compared with the simplified analytical prediction. Related works can also be found in [6], [7], [8] where notched tensile strength, fracture toughness as well as fatigue resistance for fiber/aluminium composite laminate were studied. The purpose of this work is to study the fracture toughness of a laminated material in relation to the adhesion between the layers. Load and extension were measured for a two-layer laminate specimen with a pre-crack as well as for the individual layers of the laminate. The same specimen geometry was used in all tests. For comparison, measurements were also done for the laminate without any adhesion between the layers.

\section{Materials}

Laminate in this work consists of an aluminium foil and a Low Density Polyethylene (LDPE) foil. A fully annealed AA1200 aluminium foil and LDPE with the product name LD270 is used. Load versus extension were measured for the following combinations: 1. aluminium foil with the thickness $8.98 \mu \mathrm{m}, 2$. LDPE with the thickness $27.30 \mu \mathrm{m}, 3$. Al-foil coated by LDPE giving the total thickness $36.28 \mu \mathrm{m}$ and 4. aluminium foil and LDPE joined together without adhesion between the layers and the total thickness is $36.28 \mu \mathrm{m}$. For the 3:rd case pieces of aluminium foil were cut from a roll of fabricated material. The laminate was then prepared in a Haake film extruder with a $36 \mu \mathrm{m}$ Polyester (PET) as carrier. The foil was mounted on the PET carrier while LDPE was extruded and coated on aluminium foil at a melting temperature of $278{ }^{\circ} \mathrm{C}$. A nip with the pressure 202 bar was used to press the layers together. The laminated specimen for case 3 was then cut from roll including PET, aluminium foil and LDPE. After producing the material for case 3, LDPE was continuously extruded on the PET carrier under the same conditions but without the aluminium foil. By peeling off the PET carrier, the LDPE produced here was used for making the specimen for cases 2 and 4 . Finally the specimen for case 1 is taken from the aluminium foil roll of the same direction as specimens for all other cases. Figure 1 a. shows schematically the geometry used for all cases 1 to 4 . 
The material properties are taken from [1] and [2]. To simplify the analyses the materials are approximated as perfectly plastic with the following properties for the aluminium foil, the modulus of elasticity $E=34 \mathrm{GPa}$ and the yield stress $\sigma_{0}=49 \mathrm{MPa}$. For the LDPE the modulus of elasticity was assumed to be $E=136 \mathrm{MPa}$ with the yields stress $\sigma_{0}=2 \mathrm{MPa}$. Poisson's ratio, $v$, was not measured. Here it is, for both materials, assumed to be $v=0$. Both materials are known to have a fairly high fracture toughness in plane strain so that fracture occur in plane stress for the thicknesses used here.

\section{The Experiment}

Centre cracked panels, as shown in Fig.1 a, are used for evaluating the fracture toughness of the laminated composite and components of it. Pre-fabricated cracks are manually cut, using a razor blade, to a total slit length of $2 a=45 \mathrm{~mm}$. The width and gauge length of the specimens was $2 W=95 \mathrm{~mm}$ and $2 h=230 \mathrm{~mm}$, respectively. A pair of wide clamps is utilised as shown in Fig. $1 \mathrm{~b}$. The tests are made in a MTS Universal Testing Machine. The upper clamp is attached to a $2.5 \mathrm{kN}$ load cell as well as a crosshead in the MTS-machine. Since the specimen is mounted vertically, the clamps are equipped with needles to facilitate a correct positioning. After the positioning of a sample the upper and lower clamps are closed and the pressure is applied by tightening four equally spaced quick-acting locking nuts along the front of each clamp as Fig. $1 \mathrm{~b}$ shows. Locking pins at the centre of the front jaws keep the clamps in an open position during mounting. Specimens are tested by traversing the upper crosshead up to move the sample under increasing tension $u$ at a constant crosshead speed of $9.2 \mathrm{~mm} / \mathrm{min}$. The software TestWorks is used to control the load frame and also to record data. During testing both displacement between the crossheads and load is monitored and recorded. The laminate tests are run until the entire cross section has fractured.

The experimental result is collected in Fig. 2. The differences between the load carrying capacity of the laminate and that of the components are surprisingly large. The peak force for the aluminium foil is $13 \mathrm{~N}$ and is reached when the foil is extended a little less than $1 \mathrm{~mm}$. At that extension the LDPE-foil carries not more than $1 \mathrm{~N}$. Still the composite of the to foils carries around $20 \mathrm{~N}$ at the same extension. When extended $2 \mathrm{~mm}$ the laminate carries around $23 \mathrm{~N}$ whereas the LDPE foil alone, carries $2 \mathrm{~N}$ and the aluminium foil does not contribute at all while being fractured. which means that the load carrying capacity i multiplied 10 times.

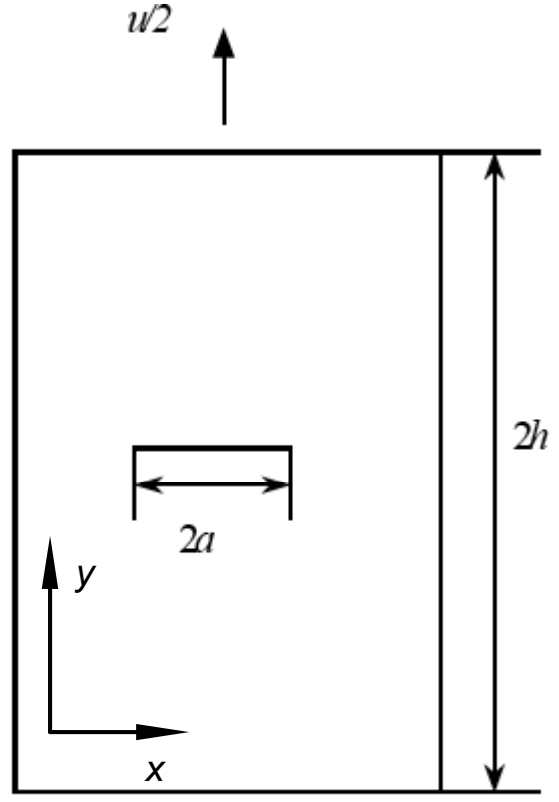

a.

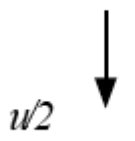

b.

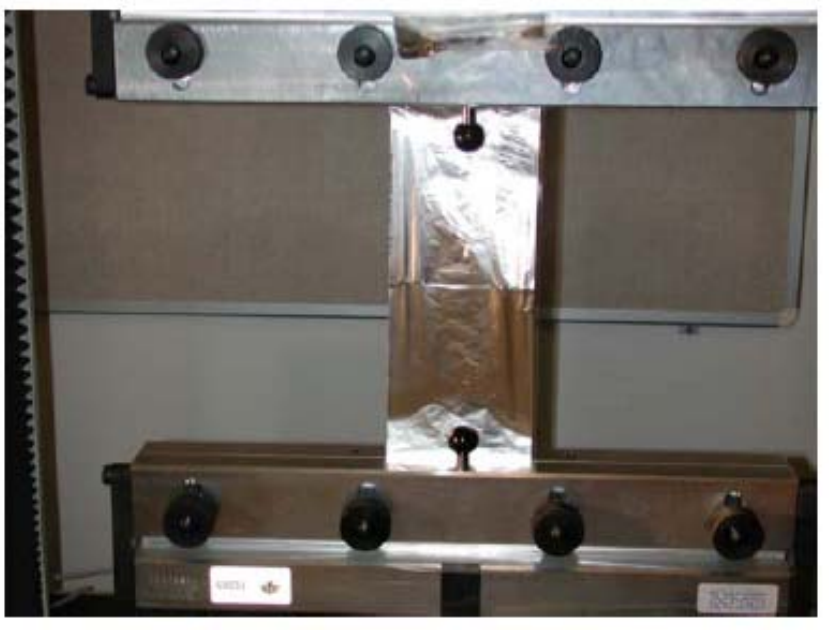

Figure 1 a. Test specimen geometry as a centre cracked panel. 2. Experimental setup for the tensile test. 


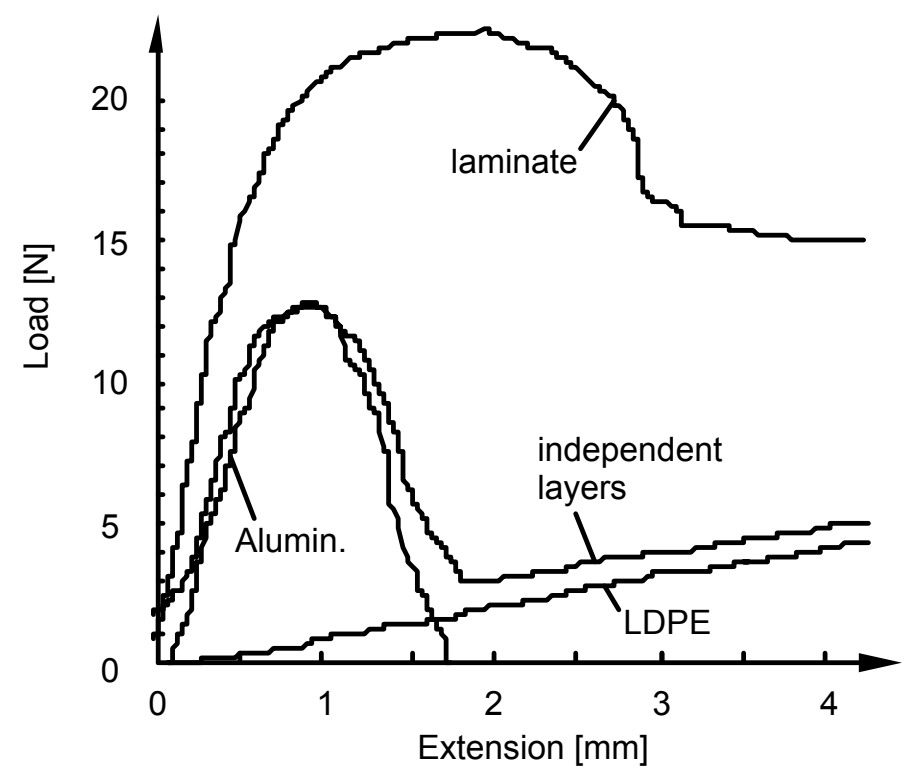

Figure 2. Load displacement curves for the laminate, including individual layers of aluminium and LDPE. Sheets of aluminium and LDPE put together with no interconnection gave the curve "independent layers".

The work done during the first $4 \mathrm{~mm}$ of elongation is more than 5 times the sum of the work done on the aluminium and the LDPE foils separately. For larger elongation the differences become less. The LDPE foil could not be conveniently tested to complete failure because of the limited motion of the grips. A rough estimate by extrapolation of the tensile test result for the LDPE suggests that the energy consumed to completely rupture the laminate equals that of the aluminium and the LDPE foils together.

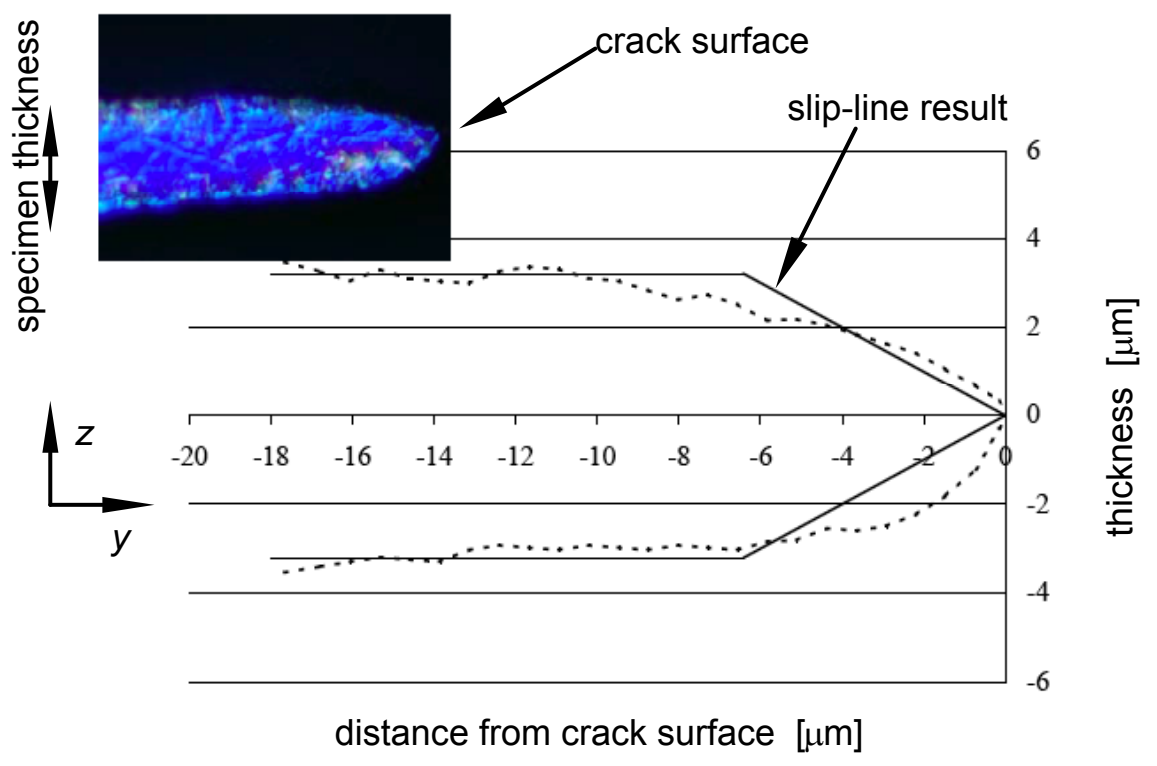

Figure 3. Cross section of the aluminium foil after fracture. The dashed curve show the mapped contour compared with the result predicted by a slip-line solution for the plastic deformation leading to fracture. The figure shows the a cross section of the lower crack surface with reference to Fig. 1 a. 
Onset of crack growth was not noted because of the difficulties give a clear definition of the phenomenon in the laminate. Early, at an elongation of less than a mm some amount of crack growth was observed in the aluminium. Crack growth with the meaning that the traction free surfaces are created is also difficult to pinpoint whereas thin ligaments of LDPE remain even after total fracture of the aluminium foil.

\section{Fracture Toughness}

Figure 3 shows a cut along the $y-z$ plane, assuming that the sheets stretching out the $x-y$ plane with the crack propagating along $x$-axis as is shown in Fig. 1 a. No traces of final rupture can be observed on the crack surfaces. This leads to the conclusion that the process leading to fracture is continuous localized straining (necking) until the cross sectional area ahead of the crack tip almost vanishes. The assumption here is that the final fracture of the remaining ligament between the upper and the lower crack surfaces has little influence on the toughness of the sheet. The fracture process can be explained within the framework of continuum mechanics and there is not any need for a fracture criterion. Assuming that the material is perfectly plastic, immediately as the yield stress is reached strains localize. Cross sectional slip as shown in Fig. 4 a proceeds as the width, $s$, of thinnest section depend on elongation of the sheet. The stress in the thinner section is uniform and equals $4 / \sqrt{ } 3 \sigma_{0}$. Therefore the elastic strain in the specimen is $2 / \sqrt{3} \sigma_{0}(s / t)\left[\left(1-v^{2}\right) / E\right]$, where $t$ is the original thickness of the specimen. The following is then readily realized

$$
s=(t-u) /\left[1-(2 / \sqrt{3})\left(1-v^{2}\right) \sigma_{o} h /(t E)\right]
$$

where $u$ is the total elongation and $h$ is the hight of the specimen. The dissipated work per unit of thickness of the system in Fig. 4 becomes $(1 / \sqrt{ } 3) t \sigma_{0}$. This equals the critical driving force, $K_{c}^{2} / E$, to extend the crack under plane stress conditions. Thus, the fracture toughness, $K_{c}$, is obtained as

$$
K_{c}=\sqrt{(1 / \sqrt{3}) \sigma_{o} t E}
$$

$K_{\mathrm{c}}$ may of course be used as a fracture criterion under the assumption that the scale of yielding is small to avoid three dimensional calculation of the fracture processes.

\section{Numerical Analyses}

The behaviour of the laminate was computed using a finite element method. A strip with the length 1.5 times the height, $t$, is pulled at its ends pulled to the length 2.25t. The strip is covered with 442 8-node isoparametric elements with reduced integration. The height of the strip is reduced $0.25 \%$ at the centre or the specimen to avoid that the strains localize somewhere else. The code uses an updated Lagrangian scheme and has the capability to compute the deformation until the hight becomes a tenth of it original size. After that converging solutions are still obtained but the reduced flexibilty because of the extremely elongated elements prevent further thinning of the strip.

To quallify the model, the homogeneous materials, i.e. aluminium and LDPE was first computed. The results where convincing with less than $6 \%$ under estimation of the maximum force and a fairly accurate estimation elongation at disappearing cross section of around $0.95 t$. When both materials are yielding the total force is denoted $F_{\mathrm{m}}$. This is used to normalize the result as is the total dissipated energy when both layers are ruptured, i.e. $t F_{\mathrm{m}} / 2$.

a.

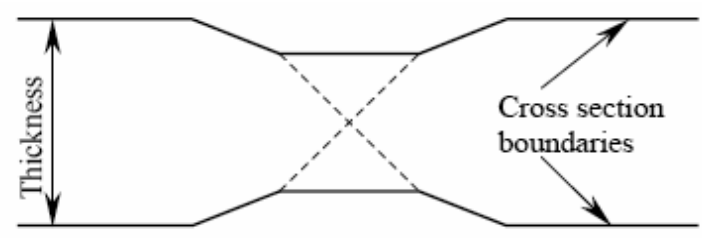

b.

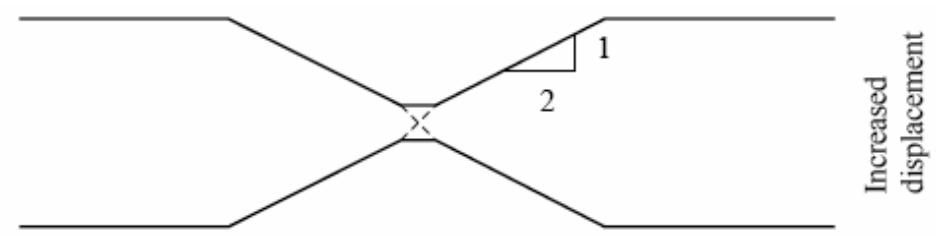

Figure $4 \mathrm{a}$ and $\mathrm{b}$. The progress of cross sectional slip along $45^{\circ}$ slip-planes produce a characteristic 1:2 slope of the crack surface 
Figure 5. Localized straining of a laminate strip a. with perfectly bonding interface and $b$. with some amount of delamination
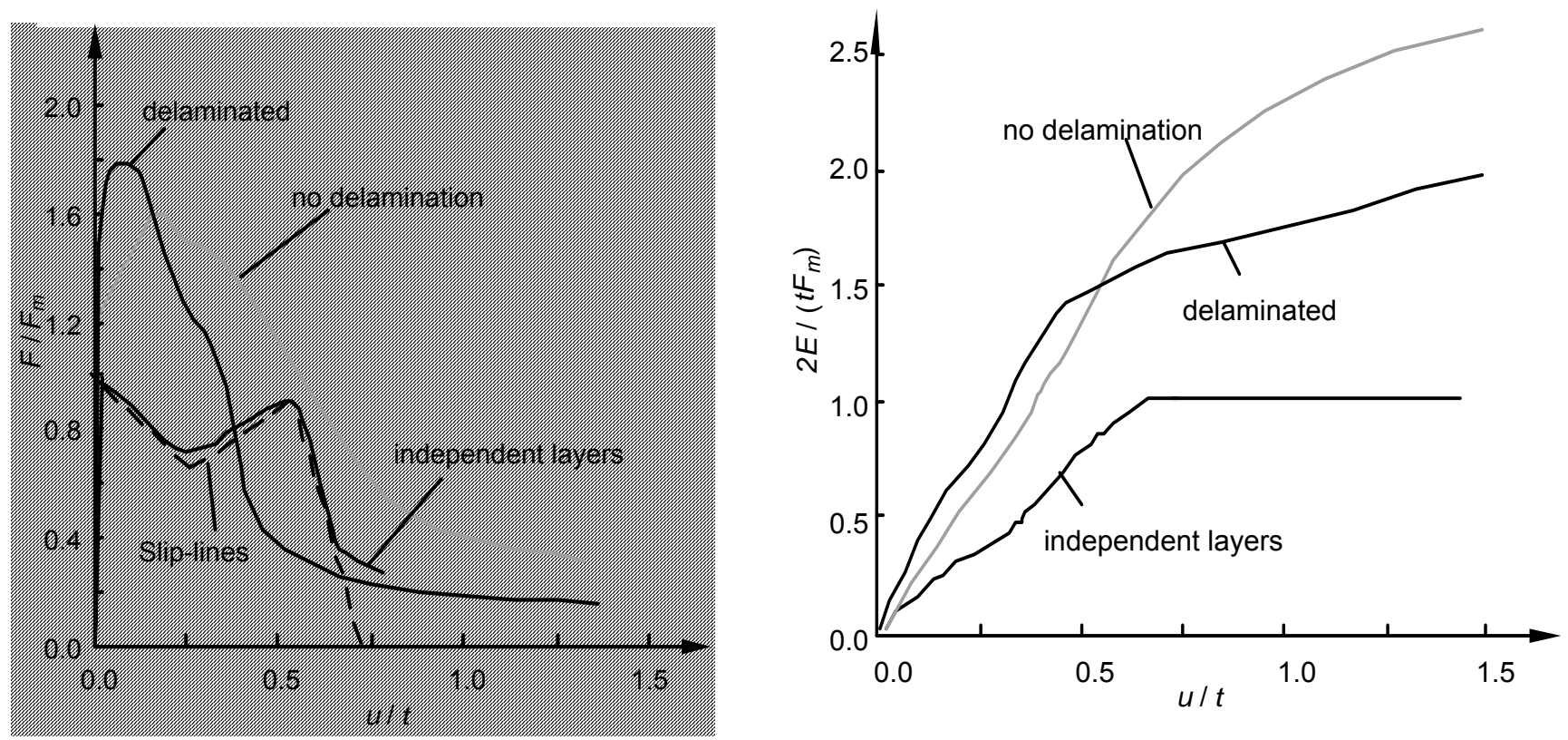

Figure 6 a. Normalized force versus normalized dislacement, b. Normalized energy dissipated in the fracture process region.

Figs. $5 \mathrm{a}$ and $\mathrm{b}$ shows the deformation of two computed geometries. The top layers have the properties of the aluminium with $a$ smaller ratio $\sigma_{0} / E$ than the lower layer (LDPE). Therefore the aluminium part first become plastic. As observed in Fig. $5 \mathrm{a}$, this introduce very large strains also in the In LDPE layer. This may be the reason for the large dissipation observed during the experiment and in the simulation as Fig. 6 a and b shows that the energy dissipation becomes much larger than what is anticipated considering the slip-line result and the result for which for comparison also is included in Figs. $6 \mathrm{a}$ and $\mathrm{b}$.

To simulate some extent of delamination a small part of the interface between the two layers was left unbonded. This part was placed in the centre of the strip. In Fig $5 \mathrm{~b}$ the result shows that the load carrying capacity is much higher than for the two individual layers. However, dissipated energy is slightly lower than for the perfectly bonded laminate.

\section{Conclusions}

Fracture of a laminate composed of two thin layers of aluminium and LDPE is observed to occur through localized straining to seemingly vanishing cross section. The strength of the laminate is surprisingly high with regard to the strength of the component materials. The fracture process is estimated using a slip-line solution and using finite elements. Also here increased strength and a very large dissipation of energy proceed fracture of the laminate. The thin stiffer aluminium foil seems to require large straining also in the weaker LDPE layer. The effect is a large mechanical resistance in the LDPE at nominal strains for which this material provide minimal mechanical response 


\section{Acknowledgements}

This research was sponsored by The Knowledge Foundation and the companies Tetra Pak and Stora Enzo. We are grateful to Prof. M. Ristinmaa, B. Sørensen and P. J. Gustavsson for encouragement and fruitful discussions.

\section{References}

[1] Kao-Walter, S. and Ståhle, P. (2001) "In situ SEM study of fracture of an ultra thin Al-foil - modelling of the fracture process", SPIE 3rd ICEM proceeding.

[2] Kao-Walter, S., Dahlström, J. Karlsson,T. and Magnusson, A. (2002) "A study of relation between mechanical properties and adhesion level in a laminated packaging material", Mech. of Comp. Mat., Vol.39, Issue Nr.5-6, 2003.

[3 ] Lau, C. C. (1993) "A Fracture Mechanics Approach to the Adhesion of Packaging Laminates", Doc. Thesis, Imperial College of Science, UK.

[4] Tryding, J. (1996) "In Plane Fracture of Paper", Doc. Thesis, Division of Structural Mechanics, Lund Institute of Technology , Sweden.

[5] Kao-Walter, S. and Ståhle, P. (2001) "Mechanical and Fracture Properties of Thin Al-foil”, Research report, Blekinge Inst. of Tech., 2001:09.

[6] Macheret, J. and Bucci, R.J. (1993) "A crack Growth Resistance Curve Approach to Fiber/Metal Laminate Fracture Toughness Evaluation”, Eng. Frac. Mech. Vol.45, N0.6, pp.729-739.

[7] Gregory, M.A. and Roebroels, G.H.J.J. (1991) "Fiber/metal laminates: a solution to weight, strength and fatigue problems", 30th Ann. Conf. of Metallurgical Society of CIM, Ottawa, Canada.

[8] Wells, J.K. and Beaumont, P.W.R. (1987) "The prediction of R-curves and notched tensile strength for composite laminates", J. Mater. Sci. 22, 1457-1468. 